\title{
Synovial fluid level of vascular endothelial growth factor (VEGF) can predict functional status and radiological severity in patients with knee osteoarthritis (OA)
}

Aim of the work: Detection of serum and synovial fluid level of Vascular Endothelial Growth Factor (VEGF) in patients with knee osteoarthritis (OA) and to determine its relation to radiological severity and function status.

Patients and methods: Thirty OA patients and thirty controls were included. Function status was assessed using the Western Ontario and McMaster Universities Osteoarthritis Index (WOMAC). Knee pain evaluated using the Visual Analogue Scale (VAS). Serum and synovial fluid level of VEGF were measured. Kellgren-Lawrence grading scale (KL) used to assess radiological severity.

Results: The mean of patients' age was $50.33 \pm 5.3$ years ( 20 females and 10 males) with disease duration of $7.03 \pm 2.12$ years. Mean synovial fluid VEGF of OA patients was $964.1 \pm 396.72$, mean serum VEGF of OA patients was $219.1 \pm 96.95$. No difference between serum VEGF of patients and control groups $(p=0.787)$ Significant positive correlation between serum and synovial VEGF in OA patients $(r=0.58, p=0.001)$ Significant positive correlation between the synovial fluid VEGF and patients' age, disease duration, inactivity stiffness, ESR, VAS, WOMAC and KL grade scale $(p<0.0001, p=0.029, p<0.0001, p<0.0001, p=0.00$ $3, p<0.0001, p<0.0001)$. Significant positive correlation between serum VEGF and patients' age, inactivity stiffness, WOMAC, ESR and KL grade scale ( $p<0.0001, p=0.005, p=0.001, p=0.001, p=0.010)$. Synovial fluid VEGF was the significant predictors for determining the function status ( $p<0.0001)$; while for determining severity; synovial fluid VEGF, patients' age and VAS were significant predictors ( $p<0.0001, p=0.001$ and $\mathrm{p}=0.029$ respectively).

Conclusion: Serum and synovial fluid VEGF correlated with the clinical features, functional status and radiological features of Knee OA. Synovial VEGF can predict both functional impact and radiological severity in patients with knee OA.

Keywords: knee osteoarthritis (OA) • vascular endothelial growth factor (VEGF) • Western Ontario and McMaster Universities Osteoarthritis Index (WOMAC) • Kellgren-Lawrence grading scale (KL)

\section{Introduction}

Osteoarthritis (OA) is a joint disease that is accompanied by varying degree of pain, functional limitation and disability. Osteoarthritis (OA) is expected to become more common with increase age and overweight populations. It is characterized by degradation of articular cartilage, osteophytes formation, sclerosis of subchondral bone, joint capsule alterations, destruction of extracellular matrix and synovitis [1-4].

Knees are the most commonly affected joints by primary OA [5], and it was reported that radiographic progression has been detected even with early Knee OA (KOA) involvement in Egyptian patients [6,7]. Chronic inflammation accompanied by angiogenesis play an important role in the pathophysiology of osteoarthritis and affect disease progression [2,8]. Angiogenic factors such as Vascular Endothelial Growth Factor (VEGF) facilitate the extravasation of plasma and recruitment of inflammatory cell. Endochondral ossification and osteophytes formation occurs as a result of angiogenesis that occur at osteochondral junction. Angiogenesis and joint damage further exacerbate inflammation [9].

Vascular Endothelial Growth Factor (VEGF) secreted by endothelial cells is a potent proangiogenic growth factor that exerts its effects by binding to the signaling tyrosine kinase receptors VEGF receptor 1 and VEGF receptor

\author{
Rasha Ali Abdel-Magied*, \\ Hanaa AS AbuOmar, Aliaa Monir \\ Higazi, Abeer Awad \& Amal A \\ Hassan \\ Faculty of Medicine, Minia University, \\ Minia, Egypt \\ *Author for correspondence: \\ rashahazem@yahoo.com
}


$2[10,11]$. Chondrocytes within the superficial layer of the articular cartilage in human with $\mathrm{OA}$ was found to express VEGF and its receptors cartilage and accompanied by OA development and progression $[11,12]$, so VEGF in plasma and synovial fluid found to be correlated with disease severity in knee OA [13]. The study aimed to detect serum and synovial fluid VEGF level in patients with knee $\mathrm{OA}$ and to determine its relation to disease severity and function status.

\section{Patients and methods}

The study was conducted on 30 patients with OA attending the Rheumatology outpatient clinic, Minia University Hospital. All patients fulfilled the 1986 ACR criteria for diagnosis of knee osteoarthritis [14]. Thirty apparently healthy age and sex matched individuals served as a control group. Only OA patients with effusion candidate for aspiration were included.

Exclusion criteria; diabetes mellitus, other causes of arthritis, history of receiving chondroprotective drugs or systemic corticosteroid medication in the last 6 months, recent intra-articular either corticosteroid or hyalouric injection within the last 3 months, history of trauma, generalized osteoarthritis. The study protocol was approved by the local Ethics Committee of Faculty of Medicine and was in agreement to the World Medical Association Declaration of Helsinki and an informed consent was obtained from all patients. Fully history and clinical examination with special attention to the knee joints was performed for all patients. Function status was assessed using Western Ontario and McMaster Universities Osteoarthritis Index (WOMAC); for assessment of pain, stiffness and physical function of patients with knee OA. The scores are summed for items in each subscale as follows: pain $=0-20$, stiffness $=0-8$, physical function $=0-68$ and the total score of WOMAC is calculated by adding the items of the three subscales (0-96) [15]. The Visual Analogue Scale (VAS) for joint pain was evaluated [16]. Plain X-ray anteroposterior view of the knee joint on standing position to assess radiological severity using Kellgren-Lawrence grading scale [17].

Laboratory investigation included CBC, ESR, C-Reactive Protein (CRP) and Vascular Endothelial Growth Factor (VEGF) was quantitively assessed in the serum and synovial fluid of OA patients while in control it was measured in serum only using human VEGF Picokine Enzyme Linked Immunosorbent Assay (ELISA) Kit (BOSTER BIOLOGICAL
TECHNOLOGY Co., ltd). Synovial fluid aspiration: from OA patients was aspirated for assessment of VEGF in synovial fluid. Synovial fluid was not aspirated from controls for ethical considerations, and we followed the previous published researches for synovial fluid level of VEGF in healthy joints. The reference value for synovial fluid VEGF in healthy joints was taken after Fay et al. the median VEGF level was 36 $\mathrm{pg} / \mathrm{ml}$ (range 12-92 pg/ml) [18].

Statistical analysis: Data were analyzed by the statistical package for the social sciences (SPSS Inc., Chicago, IL, USA) version 16.0. Categorical and quantitative variables were respectively described as numbers/percentage (\%) and mean \pm SD. Variables were compared by Mann Whitney or Chi-squared tests. Analysis of Variance test (ANOVA or F-test) is used for comparison of more than 2 means. Correlation between variables was calculated by Spearman rho correlation coefficient. Regression analysis was performed to find predictors of functional disability and disease severity and p-values 0.05 were considered significant.

\section{Results}

Characters of OA patients, function status, laboratory parameters and radiological grading are presented in Table 1. Serum and synovial fluid levels of VEGF in OA patients and control (Figure 1); In the control the mean ESR was 7.36 $\pm 1.93 \mathrm{~mm} / 1^{\text {st }}$ hour, the mean serum VEGF of controls $211 \pm 107.86 \mathrm{pg} / \mathrm{ml}$. No difference between serum VEGF of patients and control groups $(p=0.787)$. Serum and synovial fluid levels of VEGF in different OA grading's are presented in Table 2. Mean serum and synovial levels of fluid VEGF in OA grading (Figure 1). Correlation between serum and synovial fluid levels of VEGF with demographic, clinical, functional assessment score, VAS, laboratory data and radiological grading of OA patients are presented in Table 3 and Figure 2. There was positive significant correlation between serum and synovial VEGF in patients with OA group ( $p=0.001, r=0.58$ ) (Figure 2 ). Significant positive correlation between synovial VEGF and WOMAC pain $(\mathrm{r}=0.368, \mathrm{p}=0.045)$, WOMAC stiffness $(\mathrm{r}=0.401, \mathrm{p}=0.028)$ and WOMAC function $(r=0.518, p=0.003)$.

Among the many independent factors including the serum and synovial fluid VEGF levels, patients' age, VAS and Kellgren-Lawrence grading in determining the function status (using WOMAC as dependent factor), only synovial 


\begin{tabular}{|c|c|c|}
\hline \multicolumn{2}{|c|}{ Parameter } & OA patients \\
\hline \multicolumn{2}{|c|}{ mean $\pm S D$ and/or $n(\%)$} & $\mathrm{N}=30$ \\
\hline \multicolumn{2}{|c|}{ Age (years) } & $50.33 \pm 5.30$ \\
\hline \multicolumn{2}{|c|}{ Gender F:M } & $20: 10$ \\
\hline \multicolumn{2}{|c|}{ Disease duration (years) } & $7.03 \pm 2.12$ \\
\hline \multirow{4}{*}{ Functional assessment } & Total WOMAC & $70.7 \pm 13.89$ \\
\hline & WOMAC Pain & $13.56 \pm 5.26$ \\
\hline & WOMAC Stiffness & $5.26 \pm 1.04$ \\
\hline & WOMAC Physical function & $51.2 \pm 11.1$ \\
\hline \multicolumn{2}{|c|}{ VAS pain } & $5.36 \pm 1.13$ \\
\hline \multirow{6}{*}{ Musculoskeletal manifestations } & morning stiffness (minutes) & $17 \pm 9.79$ \\
\hline & inactivity stiffness (minutes) & $19.33 \pm 5.04$ \\
\hline & Deformity & $9(30)$ \\
\hline & Instability & $4(13.3)$ \\
\hline & $\mathrm{ACL}$ tear & $3(10)$ \\
\hline & MCL tear & $1(3.3)$ \\
\hline \multirow{5}{*}{ Radiological grading } & \multicolumn{2}{|c|}{ Kellgren-Lawrence grading scale } \\
\hline & Grade 1 & 0 \\
\hline & Grade 2 & $14(46.7)$ \\
\hline & Grade 3 & $10(60)$ \\
\hline & Grade 4 & $6(20)$ \\
\hline \multirow{3}{*}{ Laboratory investigations } & $\mathrm{ESR} \mathrm{mm} / 1^{\mathrm{st}} \mathrm{hr}$ & $12.99 \pm 1.19$ \\
\hline & Serum VEGF (pg/ml) & $219.1 \pm 96.95$ \\
\hline & Synovial fluid VEGF (pg/ml) & $964.1 \pm 396.72$ \\
\hline
\end{tabular}

OA: Osteoarthritis; VAS: Visual Analogue Scale; WOMAC: Western Ontario And Mcmaster Universities Osteoarthritis Index; ACL: Anterior Cruciate Ligament; MCL: Medial Collateral Ligament; ESR: Erythrocyte Sedimentation Rate; VEGF: Vascular Endothelial Growth Factor
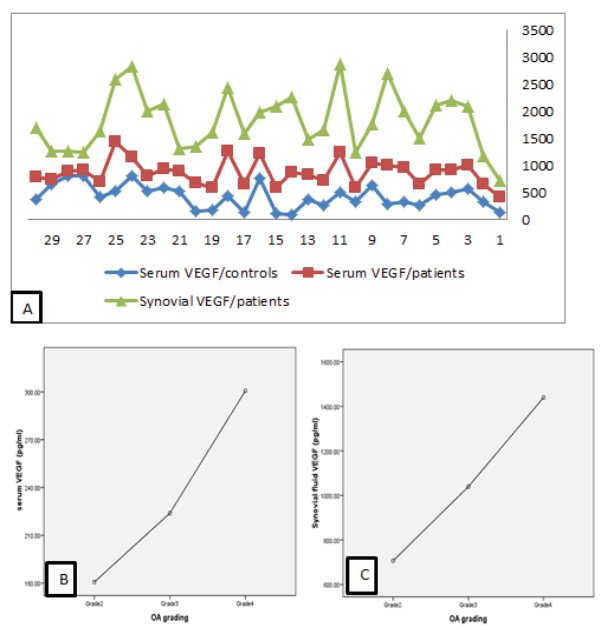

Figure 1. ( A) Serum and synovial VEGF levels in OA patients and control (B) Mean serum VEGF level in different OA grading (C) Mean synovial fluid VEGF level in OA grading.

fluid VEGF was the significant predictors ( $\mathrm{p}$ 0.0001); while for determining severity (using Kellgren-Lawrence grading score as dependent factor) among many independent factors including the serum and synovial fluid levels of VEGF, patients' age, VAS and WOMAC; synovial fluid VEGF levels, patients' age and VAS were significant predictors $(\mathrm{p} 0.0001, \mathrm{p}=0.001$ and $\mathrm{p}=0.029$ respectively).

\section{Discussion}

Osteoarthritis (OA) is a degenerative joint disease characterized by degradation of the articular cartilage, sclerosis of subchondral bone, formation of osteophyte and inflammation of the synovial membrane [19]. Vascular endothelial growth factor was up-regulated in osteoarthritis and was found to be expressed in the articular cartilage and in the osteophytes within the hypertrophic chondrocytes of OA patients which indicate the role of VEGF in angiogenesis during osteophyte development in OA [20,21].

In the current study serum VEGF level was higher in $\mathrm{OA}$ patients than that of healthy controls, without significant difference in comparison between OA patients and controls $(\mathrm{p}=0.787)$. These results were in agreement with the study of Lee et al. [22] who studied 49 OA patients, and 80 normal controls. The mean serum VEGF concentration was higher in OA patients in comparison to controls with no difference between OA patients and controls. In 


\begin{tabular}{|c|c|c|c|c|c|}
\hline \multicolumn{7}{|c|}{ Table 2. Serum and synovial fluid VEGF levels in different grades of knee OA. } \\
\hline ParameterMean \pm SD & KL grade 2 (KL2) & $\begin{array}{c}\text { KL grade 3 } \\
\text { (KL3) }\end{array}$ & $\begin{array}{c}\text { KL grade 4 } \\
\text { (KL4) }\end{array}$ & F & p value \\
\hline Serum VEGF (Pg/ml) & $180.71 \pm 83.43$ & $223.9 \pm 91.68$ & $\begin{array}{c}300.67 \pm \\
95.56\end{array}$ & 3.874 & 0.033 \\
\hline Synovial fluid VEGF (Pg/ml) & $706.5 \pm 283.57$ & $\begin{array}{c}1039.4 \pm \\
293.2\end{array}$ & $\begin{array}{c}1439.7 \pm \\
282.4\end{array}$ & 14.236 & $<0.0001$ \\
\hline \multicolumn{2}{|c|}{ KL: Kellgren-Lawrence; VEGF: Vascular Endothelial Growth Factor } \\
\hline
\end{tabular}

\begin{tabular}{|c|c|c|}
\hline Parameters $r(p)$ & Serum VEGF & Synovial fluid VEGF \\
\hline Age of the patients & $0.63(0.0001)$ & $0.60(0.0001)$ \\
\hline Duration of the disease & $0.48(0.007)$ & $0.40(0.029)$ \\
\hline Age of disease onset & $0.54(0.002)$ & $0.42(0.022)$ \\
\hline Morning stiffness duration & $0.13(0.500)$ & $0.22(0.245)$ \\
\hline Inactivity stiffness duration & $0.50(0.005)$ & $0.60(0.0001)$ \\
\hline WOMAC & $0.59(0.001)$ & $0.63(0.0001)$ \\
\hline ESR & $0.56(0.001)$ & $0.77(0.0001)$ \\
\hline VAS & $0.351(0.058)$ & $0.52(0.003)$ \\
\hline $\mathrm{KL}$ grade scale & $0.47(0.01)$ & $0.72(0.0001)$ \\
\hline
\end{tabular}

WOMAC: Western Ontario And Mcmaster Universities Osteoarthritis Index; ESR: Erythrocyte Sedimentation Rate; VAS: Visual Analogue Scale; KL Grade Scale: Kellgren-Lawrence Grading Scale; VEGF: Vascular Endothelial Growth Factor; Significant $\mathrm{p}$ Value $<0.05$
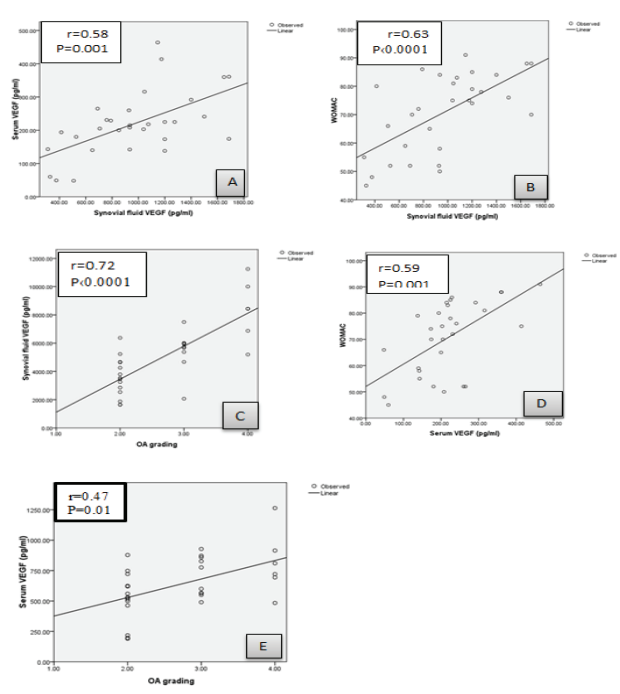

Figure 2. ( A) Correlation between synovial fluid VEGF and serum VEGF of OA patients (B) Correlation between synovial VEGF of OA patients and WOMAC (C) Correlation between synovial VEGF and KL grading scale of $O A(D)$ Correlation between serum VEGF of OA patients and WOMAC (E) Correlation between serum VEGF and KL grading scale of $O A$.

addition Kim et al. [23] studied 34 OA patients and 36 healthy controls and reported that no difference between serum VEGF in OA patients in comparison to healthy controls. Seatan et al. [24] who studied $80 \mathrm{OA}$ and 20 controls and reported no significance between plasma VEGF level in patients and controls but plasma level was lower in OA patient compared to control.
However, Mabey et al. [2] studied 31 OA patients compared to 15 healthy controls and they concluded that plasma level of VEGF was significantly higher in OA plasma than control (p 0.001).

Synovial fluid level of VEGF was significantly higher in OA patients in comparison to paired serum levels and it was significantly higher when compared to the reference value $[18,25]$. In agreement with our study, Seatan et al., [24] who reported that level of VEGF in synovial fluid of patients with knee $O A$ was tenfold higher than plasma level (p 0.001). Also Gaballah et al. [3] who studied 20 patients with primary knee OA, they reported a statistically significant increase in the level of synovial VEGF in patients when compared to the reference value of the level of synovial VEGF ( $\mathrm{p}=0.0001)$.

Our study revealed a significant correlation between synovial fluid VEGF with patients' age, duration of the knee OA and VAS scale for knee pain. There was significant positive correlation between synovial VEGF and total WOMAC, WOMAC pain, stiffness and function. There was positive correlation between serum VEGF and total WOMAC.

In agreement with our study Gaballah et al. [3] detected significant correlation between synovial fluid VEGF and the duration of knee pain, VAS 

status and radiological severity in patients with knee osteoarthritis (OA)

of knee pain and total WOMAC score.

Rübenhagen et al. [26] detected significant correlation between the synovial fluid level of VEGF and the age of patients. On the contrary Kim et al. [23] found negative correlation between synovial fluid VEGF and total WOMAC, WOMAC stiffness and WOMAC function. Also there was negative correlation between synovial VEGF and total WOMAC, WOMAC pain and WOMAC stiffness.

In our study the mean value of synovial fluid level of VEGF was higher in patients with KL grade 4 than those of KL grade 2. Serum and synovial fluid levels of VEGF exhibited a positive correlation with the radiographic severity of knee OA.

In agreement with our study Kim et al. [23], they found that the level of VEGF was higher in patients with $\mathrm{KL}$ grade 4 than those with KL grade 2 and there was positive correlation between synovial VEGF and $\mathrm{KL}$ grades. In addition, Lee et al. [27] who studied 34 OA patients, they found that the synovial fluid level of VEGF was higher in patients with KL grade 4 than those of KL grade 2 and synovial VEGF level was positively correlated with KL scores, however they differ from our study as they found no significant difference in plasma VEGF level among OA patients with different KL grades.

Also in agreement of our study Mabey et al. [2], who assessed OA severity using the KellgrenLawrence (KL) grading score, they concluded that synovial fluid VEGF level in OA patients was positively correlated with the radiographic severity of knee $\mathrm{OA}$ and plasma VEGF significantly increased in advanced OA patients. In addition Seatan et al. [24] found that plasma and synovial fluid levels of VEGF showed a positive correlation with the radiographic severity of knee OA. Also in agreement with our study Jian-lin et al. [28], Ding et al. [29] concluded that level of VEGF was significantly higher and positively correlated with the radiographic severity in OA patients. Rübenhagen et al. [26] who examined synovial fluid aspirated from 82 OA patients, who had undergone surgery for total knee replacement, they found that the level of VEGF in the synovial fluid was correlated with the radiological grading as assessed by Kellgren and Lawrence score. In the study of Klaus et al. [30], they found a significant correlation between level of VEGF in synovial fluid and radiographic damage of knee OA, however they used Ahlbäeck score. In contrast to these results,
Anitua et al. [31] who did not find a significant association between radiographic grading (using Ahlbäck score) and the level of VEGF.

Among the study limitations is the small number and corss-sectional study design. A study on a larger number of patients could better delineate the findings and understanding of the role of VEGF on the development and progression of osteoarthritis to help finding the therapeutic targets for prevention and treatment of OA.

In conclusion, serum and synovial fluid VEGF correlated with the clinical features, functional status and radiological features of Knee OA. Synovial VEGF can predict both functional impact and radiological severity in patients with knee OA.

\section{Conflict of interest}

None

\section{Funding}

This research did not receive any specific grant from funding agencies in the public, commercial, or not-for-profit sectors.

\section{References}

1. El-Najjar AR, Ezzeldin N, Khalil SS et al. Vascular endothelial growth factor and colour doppler ultrasonography in knee osteoarthritis: Relation to pain and physical function. Egypt. Rheumatol. (2018).

2. Mabey T, Honsawek S, Saetan N et al. Angiogenic cytokine expression profiles in plasma and synovial fluid of primary knee osteoarthritis. International Orthopaedics 38(9), 1885-1892 (2014).

3. Gaballah A, Hussein NA, Risk $M$ et al. Correlation between synovial vascular endothelial growth factor, clinical, functional and radiological manifestations in knee osteoarthritis. Egypt. Rheumatol. 38(1), 29-34 (2016).

4. Hussein NA, Sharara G. Correlation between serum leptin, cytokines, cartilage degradation and functional impact in obese knee osteoarthritis patients Egypt. Rheumatol. 38(2), 117-122 (2016).

5. Mahmoud GA, Moghazy A, Fathy S et al. Osteoarthritis knee hip quality of life questionnaire assessment in Egyptian primary knee osteoarthritis patients: Relation to clinical and radiographic parameters. Egypt. Rheumatol. (2018).

6. El-Brashy AS, El-Tanawy RM, Hassan WA et al. Potential role of vitamin $\mathrm{K}$ in radiological progression of early knee osteoarthritis patients. Egypt. Rheumatol. 38(3), 217-223 (2016). 
7. Nawito ZO, El-Azkalany GS, El-Sayad M. Nottingham health profile assessment of health-related quality of life in primary knee osteoarthritis patients: Relation to clinical features and radiologic score. Egypt. Rheumatol. 40(4), 265-268 (2018).

8. Sellam J, Berenbaum F. The role of synovitis in pathophysiology and clinical symptoms of osteoarthritis. Nat. Rev. Rheumatol. 6(11), 625635 (2010).

9. Bonnet CS, Walsh DA. Osteoarthritis, angiogenesis and inflammation. Rheumatology (Oxford) 44(1), 7-16 (2004).

10. Hoeben A, Landuyt B, Highley MS et al. Vascular endothelial growth factor and angiogenesis. Pharmacol. Rev. 56(4), 549-580 (2004).

11. Lingaraj K, Poh CK, Wang W. Vascular endothelial growth factor (VEGF) is expressed during articular cartilage growth and re-expressed in osteoarthritis. Ann. Acad. Med. Singapore. 39, 399-403 (2010).

12. Honsawek S, Yuktanandana P, Tanavalee A et al. Correlation between plasma and synovial fluid basic fibroblast growth factor with radiographic severity in primary knee osteoarthritis. Int. Orthop. 36(5), 981-985 (2012).

13. Liao W, Li Z, Wang $\mathrm{H}$ et al. Proteomic analysis of synovial fluid: insight into the pathogenesis of knee osteoarthritis. Int. Orthop. 37(6), 10451053 (2013)

14. Altman R, Asch E, Bloch D et al. Development of criteria for the classification and reporting of osteoarthritis. Classification of osteoarthritis of the knee. Diagnostic and Therapeutic Criteria Committee of the American Rheumatism Association. Arthritis. Rheum. 29(8), 1039-1049 (1986).

15. Ebrahimzadeh $\mathrm{MH}$, Makhmalbaf $\mathrm{H}$, Birjandinejad A et al. The western ontario and mcmaster universities osteoarthritis index (WOMAC) in persian speaking patients with knee osteoarthritis. Arch. Bone. Jt. Surg. 2(1), $57-$ 62 (2014).

16. Bruce B, Fries J. The Stanford health assessment questionnaire: a review of its history, issues, progress, and documentation. J. Rheumatol. 30(1), 167-178 (2003).

17. Kellegren JH, Lawrence JS. Radiological assessment of osteo-arthrosis. Ann. Rheum. Dis. 16 (4), 494-502 (1957).

18. Fay J, Varoga D, Wruck J et al. Reactive oxygen species induce expression of vascular endothelial growth factor in chondrocytes and human articular cartilage explants. Arthritis. Res. Ther. 8(6), 189 (2006).

19. Krasnokutsky S, Attur M, Palmer G et al. Current concepts in the pathogenesis of osteoarthritis.
Osteoarthr. Cartil. 16(3), 51-53 (2008).

20. Enomoto H, Inoki I, Komiya $\mathrm{K}$ et al. Vascular endothelial growth factor isoforms and their receptors are expressed in human osteoarthritic cartilage. Am. J. Pathol. 162(1), 171-181 (2003).

21. Bosco MC, Delfino S, Ferlito F et al. The hypoxic synovial environment regulates expression of vascular endothelial growth factor and osteopontin in juvenile idiopathic arthritis. $J$. Rheumatol. 36(6), 1318-1329 (2009).

22. Lee SS, Joo YS, Kim WU et al. Vascular endothelial growth factor levels in the serum and synovial fluid of patients with rheumatoid arthritis. Clin. Exp. Rheumatol. 19(3), 321-324 (2001).

23. Kim HR, Lee JH, Kim KW et al. The relationship between synovial fluid VEGF and serum leptin with ultrasonographic findings in knee osteoarthritis. Int. J. Rheum. Dis. 19(3), 233-240 (2016).

24. Saetan N, Honsawek S, Tanavalee A et al. Relationship of plasma and synovial fluid vascular endothelial growth factor with radiographic severity in primary knee osteoarthritis. Int Orthop. 38(5), 1099-1104 (2014).

25. Costa C, Incio J, Soares R. Angiogenesis and chronic inflammation: cause or consequence? Angiogenesis. 10(3), 149-166 (2007).

26. Rübenhagen R, Schuttrumpf JP, Sturmer KM et al. Interleukin-7 levels in synovial fluid increase with age and MMP-1 levels decrease with progression of osteoarthritis. Acta. Ortho. 83(1), 59-64 (2012).

27. Lee SH, Kim HR, Kim HY. Synovial fluid vascular endothelial growth factor could predict progression of osteoarthritis according to radiological and ultrasonographic findings. Arthritis. Rheum. 65(1), 482 (2013).

28. Jian-lin Z, Hong-song F, Hao $\mathrm{P}$ et al. The relationship between HIF-2 $\alpha$ and VEGF with radiographic severity in the primary osteoarthritic knee. Med. J. 57(3), 735-740 (2016).

29. Ding J, Niu X, Su Y et al. Expression of synovial fluid biomarkers in patients with knee osteoarthritis and meniscus injury. Exp. Ther. Med. 4(2), 1609-1613 (2017).

30. Klaus D, Matziolis G, Müller $M$ et al. In vivo imaging of human synovial microcirculation in knee osteoarthritis: interaction of microvascular dysfunction, matrix degradation and osteoarthritis progression. Dtsch. Ges. Fur. Chir. 37, 19-22 (2008).

31. Anitua E, Sánchez $M$, de la Fuente $M$ et al. relationship between investigative biomarkers and radiographic grading in patients with knee osteoarthritis. Int. J. Rheumatol. 2009, 1-4 (2009). 